\title{
Requerimientos para laboratorios de ingeniería industrial; su infraestructura, software y metodología de aprendizaje
}

\author{
Ing. Heylin Díaz Jiménez \& \& Ing. Maribel Jiménez Fernández ${ }^{2}$ \\ 1. Encargada de Cátedra Gestión de Calidad. Universidad Estatal a Distancia; e-mail: hdiaz@uned.ac.cr \\ 2. Encargada de Carrera Ingeniería Industrial. Universidad Estatal a Distancia; e-mail: mjimenez@uned.ac.cr
}

Recibido: 23 abril 2017

\section{RESUMEN}

En el presente artículo se exponen los requerimientos en cuanto a la infraestructura, software y metodologías de aprendizaje de los laboratorios especializados para la carrera de Ingeniería Industrial, basándose en lo observado en diversas universidades visitadas por una comisión institucional. La revisión de la literatura en este sentido dotará del contenido teórico y referencial en cuanto a la pertinencia y necesidad del uso de laboratorios en la carrera, de igual forma, fundamentan las estrategias de aprendizaje más apropiadas en la didáctica en el laboratorio. El laboratorio permite la experimentación por parte del estudiante, por lo que se deben hacer uso de metodologías constructivistas por ejemplo: el aprendizaje basado en resolución de problemas.

Palabras claves: laboratorios especializados, software, estrategias de aprendizaje en laboratorios, infraestructura de laboratorios.

\begin{abstract}
This article presents the requirements regarding the infrastructure, software and learning methodologies of specialized laboratories for the industrial engineering career, based on what has been observed in several universities visited by an institutional commission. The bibliographic review in this sense will have the theoretical and referential content as to the pertinence and necessity of the use of laboratories in the career, likewise, they base the most appropriate teaching-learning strategies in the didactics of a laboratory. The laboratory allows experimentation on the part of the student, which is why constructivist strategies should be used, for example: problem-based learning.
\end{abstract}

Key words: special laboratories, software, learning strategies in laboratories, laboratory infrastructure.
Aceptado: 12 octubre 2017

\section{Introducción}

En la visita realizada a las universidades mexicanas: Universidad Autónoma de Nuevo León, Universidad Autónoma Metropolitana y la Universidad Nacional Autónoma de México, elegidas por su trayectoria y prestigio. La visita se hace con el propósito de investigar los requerimientos físicos en cuanto a infraestructura, equipamiento, instrumentos, softwares y metodologías de aprendizaje; utilizadas en los laboratorios especializados de ingeniería industrial en estos dos ámbitos: infraestructura y estrategias de aprendizaje, de dichas universidades.

Ante la necesidad de ajustarse a los requerimientos que demanda la oferta de la carrera, la visita se realiza a universidades que imparten la carrera de Ingeniería Industrial, para obtener de fuente primaria (profesores y estudiantes) y por observación directa la información requerida y, a partir del informe presentado por los académicos, tomar decisiones técnica y metodológicamente válidas respecto al grado de necesidad de contar con equipos e instrumentos similares, así como la conveniencia de utilizar metodologías similares o los ajustes que se requerirían hacer para que sean pertinentes y funcionales para la modalidad de formación a distancia de la UNED y para la formación del Ingeniero Industrial que plantea la propuesta formativa del plan de estudios.

En el plan de estudios se especifica que la función principal de todo ingeniero industrial, es la mejora de los procesos productivos, mediante el ensayo experimental. La generación de criterios que permiten realizar dichos procesos, 
mismos que orientan en la toma de decisiones para el cambio de éstos procesos (Rosales, 2011). Ahora bien, según especialistas en el campo educativo de Ingeniería, es mediante el análisis y solución de problemas, logística, tecnología, métodos de investigación y estadística (UNED, 2013), aplicadas en los laboratorios de las diferentes asignaturas, como cada estudiante forma y desarrolla las habilidades requeridas como profesional en Ingeniería Industrial.

En consecuencia, el plan de estudios de la carrera cuenta con 14 asignaturas que requieren de algún tipo de laboratorio (virtual o presencial), ya sea como un medio para llevar a cabo prácticas científicas o como un complemento a una asignatura de contenido teórico. Además, se busca subsanar lo expuesto por empleadores, respecto a la deficiencia en el aprendizaje y desempeño de estos profesionales para la aplicación y puesta en práctica de los contenidos en los contextos laborales, de forma integral, (UNED, 2013).

Asimismo, las metodologías de aprendizaje de la carrera ingeniería industrial se basan principalmente en la práctica y se fundamentan en el enfoque cognitivo-constructivista (UNED, 2013); por lo tanto, la premisa es que el estudiantado desarrolle habilidades de autogestión que le faciliten la construcción de conocimiento requerido en ciencias básicas, estadística, diseño de experimentos, control estadístico de calidad, medición del trabajo, manufactura esbelta, y sistemas de gestión, haciendo uso efectivo y eficiente de los diferentes recursos (laboratorios y software) para la adquisición, interiorización y evidencia práctica de dichos conocimientos.

Cabe destacar que los contenidos mencionados anteriormente, requieren del uso de algún laboratorio y/o software especializado se requiere en asignaturas donde se requiere la aplicación de temáticas como la simulación, automatización, la mejora de procesos de producción de bienes o servicios, logística y ciencias básicas.

Además, según se plantea en el plan de estudios de la carrera, se requiere de una infraestructura y equipamiento que le permita al estudiantado llevar a cabo prácticas reales o simuladas, donde pueda desarrollar y poner en práctica, además de contenidos disciplinares, habilidades blandas que le permiten al estudiante la resolución de problemas, el desarrollo del pensamiento crítico, comunicación oral y escrita y relaciones interpersonales. Estas habilidades se pretenden evidenciar y evaluar en los espacios presenciales y virtuales de los laboratorios, donde el estudiantado de forma colaborativa puede ejecutar y desarrollar proyectos integradores que le permitan ponerlos en práctica, lo cual es un aprendizaje altamente valorado y de vital importancia, en el contexto nacional e internacional, para el desempeño de todo ingeniero industrial, (UNED, 2013).

Una vez se llevó a cabo está investigación en las diferentes universidades, por la representación de académicos de la carrera de Ingeniería Industrial de la Universidad Estatal a Distancia (UNED), se cuenta con las experiencias necesarias que permiten comunicar las impresiones obtenidas, las cuales constituyen el objetivo del presente artículo.

\section{Referente teórico}

Es importante para formar un criterio respecto a los laboratorios a observar, tener un conocimiento conceptual respecto a temáticas como la definición teórica de un laboratorio, las metodologías de aprendizaje que se proponen en diversos referentes teóricos, así como el uso de softwares y la infraestructura de los mismos.

En la Universidad Estatal a Distancia los laboratorios son definidos como un espacio de creación y transformación, el cual puede ser virtual o presencial. El proceso de creación y transformación por parte del estudiante le permite la comprobación y realimentación de conocimiento, a su vez éste puede ser socializado y mejorado al innovarse con la generación de ideas producto de las diversas experiencias que permite dicho proceso, (UNED, 2013).

La Universidad Autónoma del Estado de México define sus laboratorios como el espacio en el que se llevan a cabo procesos de comprensión e instrumentalización. En este espacio físico se hace uso de equipo especializado que permita el análisis físico, químico, biológico y 
mecánico de la experimentación con la naturaleza, (Ortega, 2000).

Se puede observar de las definiciones anteriores que existen laboratorios tanto físicos como virtuales. Ambos se definen como un espacio en el que se puede experimentar con el uso de instrumentos especializados. Los procesos que se derivan de la ejecución en el laboratorio se conceptualizan con la definición del experimento, el proceso para la experimentación lleva con sí la puesta en práctica de procesos mentales de mayor nivel. La finalidad de los experimentos es comprobar por medio de la práctica activa del estudiante, principios teóricos previamente comprendidos.

Con esto se conceptualiza el propósito de la práctica en los laboratorios utilizados para la formación de estudiantes, el cual corresponde a posibilitar al estudiante para que lleve a cabo experimentos que le permitan aplicar la teoría vista, (Ortega, 2000), este proceso demanda del estudiante capacidades mentales para el análisis e interpretación de los resultados del experimento, (UNED, 2013).

La didáctica en el laboratorio se define como aquellas estrategias didácticas que emplean los docentes para permitir la creación de trabajos científicos de modo que el estudiante se sienta atraído e interesado en el estudio y la comprensión de los fenómenos que los rodean, de esta forma se desarrollan y fortalecen los conocimientos conceptuales, procedimentales y actitudinales, así como las competencias científicas. Las prácticas de laboratorio es el medio apropiado para constituir una oportunidad en el desarrollo cognitivo y de motivacional del estudiantado. Al hacer uso de las prácticas de laboratorio como una estrategia didáctica, promueve la construcción del conocimiento científico por parte de los estudiantes, (Espinosa, González y Hernández; 2016).

Lugo, (2006) fundamenta la necesidad del uso de laboratorios en un espacio que fomenta la experimentación y el descubrimiento por medio de la aplicación práctica, proveyendo al estudiante de un conocimiento producto de la aplicación real, en la cual puede discriminar lo incorrecto a raíz del aprendizaje que le dejan sus propios errores. Los laboratorios representan una herramienta que se utiliza para afianzar los conocimientos que se adquieren en el proceso de aprendizaje. Además, para el desarrollo de habilidades como la comunicación oral y escrita, el liderazgo y la cooperación.

La agencia acreditadora de carreras de ingeniería Acreditation Board for Engineering and Technology (ABET), establece como uno de los requisitos para la acreditación el uso de laboratorios con el fin de combinar elementos teóricos y prácticos, detalla que una adecuada enseñanza con el uso de los laboratorios debe contemplar: las mediciones, análisis y diseño de ingeniería, así como la aplicación del método científico: el proceso lógico que lleva a resultados válidos o científicamente probados. Esta transición de los conceptos teóricos hacia la aplicación práctica se logra por medio de la experimentación que propicia los laboratorios, con el uso de estrategias como los ejercicios prácticos, el análisis de resultados experimentales, así como la comparación de resultados con las predicciones teóricas. En el laboratorio el estudiante verifica y valida el modelo que se ha propuesto. Esta práctica tan real y concreta es un componente motivacional para el estudiante, (Lugo, 2006).

Es por este motivo que dentro de las ventajas que se pueden destacar de los laboratorios, es que propicia el desarrollo de habilidades cognitivas. Producto de la experimentación que se desarrolla en el mismo por medio de la interactividad que fomenta un ambiente que simula el contacto con una situación del contexto laboral real, (Medina, Saba, Silva, \& de Guevara Durán, 2011).

Para lograr esta experimentación en la práctica diaria en los laboratorios, se debe hacer uso de estrategias de aprendizaje que propicien la misma, en este contexto, la Escuela Técnica Superior de Ingenieros Industriales de Madrid propone el aprendizaje basado en problemas (PBL por sus siglas en inglés). Lo anterior como un medio para que el estudiante desarrolle habilidades de trabajo colaborativo, toma de decisiones, análisis de contenidos, que le van a permitir resolver el problema planteado en el laboratorio de una forma activa, por su parte; el 
docente, se debe desempeñar como un motivador y mediador del proceso de evolución y conclusión del experimento por parte del estudiante. La estrategia PBL es acorde la declaración de la corriente pedagógica constructivista en la que el proceso de enseñanza está centrada en el alumno, donde su participación debe ser activa a lo largo del proceso de aprendizaje, (López, Nieto, Rodríguez, González \& Jiménez, 2011).

La aplicación de estrategias como PBL, le plantea un problema al estudiante, quien debe desagregarlo en sus partes, determinando las principales causas o soluciones, así mismo, se le demanda que identifique contenidos relevantes esto lo llevará al planteamiento de hipótesis. La prueba de sus planteamientos requiere de un proceso de reflexión y autoevaluación, lo que le permite la toma de decisiones, en su proceso de prueba y conclusión experimental, (Knowlton \& Sharp, 2003).

En la UNED las estrategias de aprendizaje se definen como "el conjunto de acciones, pasos o secuencias cognitivas, afectivas o motoras que se realizan de forma consciente y planificada para la solución de una situación de aprendizaje" (UNED, s.f., p. 38). De acuerdo a lo expuesto, se podrá asumir que las estrategias permitan por medio de la ejecución de las acciones, pasos o secuencias, ya sean: afectivas o motoras, la participación activa del estudiante, en la que busca por medio de la experimentación dar solución a un problema, en este proceso él mismo es responsable de generar su conocimiento, propiciando entonces procesos mentales de reflexión y autoevaluación, que lo llevan a tomar decisiones.

\section{Metodología}

En el año 2014 la representación de académicos de la UNED llevó a cabo la visita a tres universidades mexicanas para recopilar información necesaria en cuanto a requerimientos en infraestructura y equipamiento de los laboratorios especializados de la carrera Ingeniería industrial, así como su metodología de aprendizaje. Así, aprender de las experiencias vividas en estas universidades, de modo que en un futuro, sean consideradas en la implementación y funcionamiento de los laboratorios especializados de ingeniería industrial en nuestra institución.

Para la recolección de la información requerida y su posterior presentación en este artículo, se elaboraron instrumentos para la sistematización de esta información. La misma se dividió en la infraestructura que a su vez incluye los aspectos de los materiales y equipamiento. El otro punto en las guía de observación son las metodologías en las cuales se incluyen los software utilizados en las diversas temáticas.

La información se recopiló mediante visitas guiadas por docentes de cada una de las universidades, esto permitió recopilar información tanto de la observación como de las entrevistas realizadas a estas personas.

\section{Resultados}

Infraestructura, materiales y equipamiento

Según los académicos entrevistados, es vital que en los laboratorios se consideren aspectos que permitan la adecuada operación de estos, así como propiciar un ambiente seguro donde se delimiten los lineamientos y reglas a seguir dentro del mismo. Para esto en sus universidades se nombra un encargado de laboratorio quien representa la figura de autoridad y regulación del funcionamiento en todo lo que respecte al laboratorio, cuya función es velar por el adecuado uso de los equipos.

Además, se llevan a cabo cursos introductorios de capacitación en el adecuado uso del laboratorio, tanto para estudiantes como para tutores, donde se expone la utilidad y propósito de cada parte estructural del laboratorio, en cuanto a: códigos de colores de las tomas de corriente, adecuado uso de las tomas de gas, explica cómo se debe trabajar en las mesas de trabajo, artículos que pueden o no dejar en el laboratorio una vez que lo abandonan, acomodo de los diferentes instrumentos en los armarios, horarios y políticas para el uso del área de oficina dentro del laboratorio, adecuado uso de las bitácoras del almacén, políticas para entrega de instrumentos, uso del acceso con huella digital, puntos que indican una emergencia, uso y finalidad de la 
documentación presente en el laboratorio, manuales de los procesos, seguridad y manejo de desechos y soporte técnico para los equipos.

Dichos cursos introductorios, además de buscar establecer la normativa de la actividad en el laboratorio aseguran su adecuado funcionamiento y conformar un ambiente con procedimientos claros para el estudiantado, al darles el ejemplo de cómo se debe trabajar o cómo propiciar un ambiente seguro y de calidad.

Un aspecto mencionado, que aunque no se ha desarrollado a profundidad pero que sí fue una recomendación, es la valoración de riesgo en la implementación de los futuros laboratorios que se vayan a establecer en la institución, lo cual consiste en determinar de forma preventiva, los peligros latentes en el área de laboratorio, respecto a las áreas físicas y la metodología de funcionamiento del mismo Además de lo anterior, se consideran los aspectos que se establecen en la normativa que regula los laboratorios, tales como; la señalización, iluminación, ventilación, ergonomía, desechos, estructurales, de accesibilidad, mantenimiento correctivo y preventivo, además del dimensionamiento apto para evitar el hacinamiento.

Otro aspecto observado fue la parte estructural, ya que los laboratorios cuentan con un pequeño espacio designado como almacén, en el cual se guarda toda la instrumentación y material didáctico. Esta área permite designar un lugar específico para cada uno de los equipos y de igual forma para los materiales, tales como: folletos de prácticas, materiales con contenidos temáticos, entre otros. Asimismo, para el manejo y responsabilidad de éste espacio de almacenaje, se debe definir un laboratorista responsable del espacio y las llaves de acceso, este a su vez es supervisado por los profesores, lo que permite una amplitud de horario para la atención del mismo. La atención por parte del laboratorista permite mayor accesibilidad del estudiantado al espacio de almacenaje.

En cuanto a equipamiento e instrumentación los laboratorios especializados de ingeniería industrial; los que abarcan las temáticas de medición del trabajo, manufactura esbelta, procesos de manufactura y automatización de procesos industriales; contaban con equipos que simulan una línea de producción; en la presentación más simple. Esta es una banda transportadora donde el estudiante puede llevar a cabo el proceso de producción o ensamble de un artículo de forma manual, de la misma forma recopila los datos para la medición del trabajo. Esta metodología en un principio le permite al estudiante experimentar los retos y las problemáticas que se presentan en una línea de producción, de forma muy básica y artesanal.

\section{Metodología de aprendizaje}

La metodología de aprendizaje desarrollada para las temáticas de medición del trabajo, que además se adecúa al laboratorio de la banda transportadora por medio de poleas, se lleva a cabo mediante el ensamble de artículos conformados por juegos, que les permitirá simular un proceso de producción. Esto les facilita la determinación de los diferentes componentes del artículo en términos de planificación de los requerimientos de materiales (MRP por sus siglas en inglés), estudiarlo, determinar el método operacional para su ensamble, los tiempos para la ejecución, el puesto de trabajo y posteriormente diseñar la línea de producción para el ensamble en línea del artículo. Con todo esto se logra simular el proceso de una línea de producción.

Estas estrategias metodológicas por medio de prácticas le permiten al docente evaluar el desempeño y aplicación de los contenidos temáticos de la asignatura, así como habilidades y competencias de cada estudiante en el trabajo con los demás compañeros. En este aspecto también se puede rescatar la capacidad que desarrolla el estudiantado para la selección del método, el personal y el modelo para la optimización del proceso.

La otra presentación es un sistema de bandas transportadoras, integradas a un centro de mando computarizado, donde se programa la producción que se va a llevar acabo, para posteriormente evaluar los resultados obtenidos de las diferentes ejecuciones de dicha programación. Esto le permite a los estudiantes de forma automatizada realizar el balanceo de líneas y tomas 
de tiempos de producción. Los resultados, son almacenados en un programa para posteriormente migrar los datos a Excel y luego analizarlos en el software para desarrollar temáticas de análisis estadísticos y modificaciones a la línea de producción, con su respectiva validación científica. Este es el punto primordial en la ejecución profesional del ingeniero industrial, sus decisiones deben estar fundamentadas, de manera que pueda determinar cuál sería el riesgo que se corre, en términos de producción y costos al implementar cierto sistema de producción o servicios; así como su probabilidad de ocurrencia.

El centro de mando, también evalúa la seguridad y salud ocupacional, ya que el sistema permite monitorear por medio de una cámara la posición corporal del trabajador, en cuanto a la distribución del cuerpo en la estación de trabajo, de esta forma el estudiante puede realizar las mediciones respectivas hombre - máquina, para determinar el factor de riesgo ergonómico en el puesto.

\section{Software}

Estos laboratorios se complementan con los laboratorios de cómputo los cuales cuentan con softwares especializados para el análisis estadístico de datos, simulación, distribución, diseño de experimentos y calcular cómo suplir la demanda. Estos laboratorios deben estar contiguos a los laboratorios especializados de automatización de procesos, ya que de esta forma, se permite el acceso y la conexión directa entre el cálculo de las diferentes variables y el proceso de producción. Por ejemplo los softwares más utilizados son:

Minitab: éste software se utiliza para el análisis estadístico de datos. Esto posibilita al estudiante a determinar la variabilidad de los datos en estudio, de forma numérica y gráfica, así como la distribución de probabilidad de los datos obtenidos de una línea de producción, con lo que se identifica el algoritmo de probabilidad de ocurrencia que más se ajusta a los datos estudiados. Posterior a esto se realizan simulaciones y proyecciones. Por otro lado posee módulos en los que se puede realizar diseño de experimentos con múltiples factores, los cuales son comparados en cuanto a su nivel de influencia sobre la variable respuesta, esto le muestra al estudiante cuál es el factor que debe modificar para lograr los resultados que desea obtener en la variable respuesta.

Forecast Pro: Este programa utiliza datos históricos de la producción y de esta forma determina la línea de tendencia de la demanda, así como el algoritmo que más se ajusta al comportamiento de los datos, lo que permite hacer un pronóstico de la misma.

Lindo o Lingo: Este software le permite al estudiante determinar la ruta óptima de distribución, garantizando el menor costo.

Palisade y Win QSB: resuelve temáticas de investigación de operaciones y de planeación y control de la producción. Poniendo en práctica diferentes teorías tales como: las de colas, cadenas de Marcov, de decisiones, entre otras.

Promodel, Palisade y Flexim: Son utilizados para llevar a cabo simulaciones de líneas de producción, representando de forma gráfica la línea. Por ejemplo Flexim, permite la simulación por medio de la metodología de Montecarlo. El estudiante programa su línea de producción en el software y de forma gráfica puede observar el movimiento de los productos en las diferentes estaciones, para cada estación puede determinar el tiempo de ciclo y tiempo de espera. Esto le permite identificar los cuellos de botella.

La metodología de aprendizaje utilizada para implementar cada uno de estos softwares y las técnicas en laboratorio, responde a la corriente pedagógica constructivista y el enfoque curricular por competencias, donde el docente plantea una serie de prácticas que se desarrollan a lo largo de todas las asignaturas para darle un flujo sistemático y de conocimiento lógico a los temas y conceptos que adquiere el estudiante en el transcurso de la carrera.

El profesor en cada asignatura/laboratorio desarrolla varias prácticas que van en orden de 
complejidad de menor a mayor, concluyendo con un proyecto final que le llaman producto integrador, esta metodología se da a lo largo de toda la carrera en las diferentes asignaturas, utilizando los mismos laboratorios, pero van subiendo el nivel de complejidad a las actividades y proyectos, por lo que es indispensable una coordinación por parte de los profesores.

Además, en las prácticas de laboratorio el profesor evalúa el desarrollo de la habilidad de innovación, lo cual registra a medida que cada estudiante ejecuta las actividades. Al docente se le facilita un manual en el cual se le especifica las formas en las que el estudiante desarrolla las habilidades deseadas.

\section{Conclusiones}

En conclusión en cuanto a la información recopilada con respecto a los laboratorios especializados, en los ejes de infraestructura se establece que la misma debe obedecer a los estándares internacionales (estos responden de forma específica a cada disciplina) de cada tipo de laboratorio (química, física, biología, informática, automatización, entre otros) en cuanto a su climatización, iluminación, accesibilidad, mobiliario, manejo de desechos, conectividad, entre otros.

En cuanto a la infraestructura de los laboratorios especializados de la carrera, se deben considerar los siguientes aspectos: la adecuación del piso, la iluminación, las paredes, la climatización, tuberías, conectividad, señalización, accesibilidad, ventilación, entre otros aspectos; con el fin de que los equipos que se ubican en esta infraestructura desempeñen un adecuado funcionamiento y vida útil de acuerdo a lo indicado por el fabricante.

Para el desarrollo de las diversas temáticas y la integración de los conocimientos, es pertinente que el laboratorio de cómputo y el de métodos se ubiquen dentro de la infraestructura descrita de forma adyacente. Esto debido a que según lo observado y la descripción dada por los docentes, el estudiante tiene la oportunidad de diseñar en la laboratorio de cómputo y posteriormente observar o aplicar el diseño realizado en los equipos del laboratorio especializado.
Estrategias de

enseñanza-aprendizaje y software

El planteamiento para la conformación y utilización de los laboratorios especializados, se da en función del desarrollo de las temáticas, por lo tanto se debe hacer una definición del conocimiento que determinado equipo permite generar y en función de este hacer su selección. Es entonces que es viable la utilización de los mismos laboratorios con el mismo equipamiento para diversas carreras, considerando que las prácticas y la utilización diversificada de los mismos equipos permiten el desarrollo de las temáticas propias de cada carrera.

Esto respalda el planteamiento expuesto en cuanto a que el laboratorio se debe pensar en función a la temática y los contenidos que determinado equipamiento permite abordar. Es importante cuando se tome la decisión de hacer una inversión, pensar en todas las carreras que se verán beneficiadas con el equipamiento que se decida adquirir. Desde esta perspectiva los laboratorios cuentan con un equipamiento el cual va a ser utilizado por diversas disciplinas con el fin de hacer experimentos acordes a cada una de las temáticas que le compete a cada asignatura.

La función de estos laboratorios permite establecer la metodología de enseñanza aprendizaje la cual corresponde a los procesos en los que el estudiante ejecuta experimentos con ciertos instrumentos y en condiciones lo más cercanas a lo real de un ambiente laboral posible. Por lo tanto se deben plantear ejercicios prácticos, éstos deben estar caracterizados por ser una simulación del espacio laboral con sus dificultados y retos, de esta forma el estudiante tendrá la oportunidad de desempeñarse tal y como se le demandará en un futuro como profesional. El docente debe plantear ejercicios que le requiera al estudiante el planteamiento de un modelo desde su medición, análisis y diseño de ingeniería para posteriormente someterlo a verificación y validación. Para lo que puede hacer uso de los diversos software, mismos que permiten desarrollar las temáticas de forma secuencial, propiciando la experimentación simulada. 
De acuerdo a lo observado y a lo expuesto por los docente, esta ejecución práctica, se lleva a cabo con el uso del método científico, siguiendo las pautas rigurosas que toda experimentación requiere. Los resultados finales permiten la toma de decisiones, producto del análisis y la observación. Estos procesos de aprendizaje propician en el estudiante desarrollar habilidades como:

- El pensamiento crítico, debido a que el estudiante debe discriminar entre los resultados obtenidos la solución óptima al problema planteado.

- Resolución de problemas, por medio de la investigación y la experimentación que propicia el espacio de laboratorio, el estudiante puede dar respuesta a diversas problemáticas que se le presenten.

- Toma de decisiones, gracias al ambiente simulado del laboratorio y a la posibilidad de desarrollar cada experimento obteniendo un resultado muy cercano a la realidad y puede tomar decisiones que den solución a los problemas que se le han expuesto.

- Relaciones interpersonales, el estudiante ejecuta sus prácticas de laboratorio en compañía de sus compañeros y en conjunto con ellos, lo que requiere que se lleguen a acuerdos en cuanto a la forma de trabajo y las decisiones que se tomen en el transcurso de la ejecución del experimento; trabajo en equipo y colaborativo.

- Expresión oral y escrita, el estudiante debe exponer sus hallazgos tanto a sus compañeros como al docente una vez obtenido sus resultados y llegado a sus conclusiones, de igual forma, estos resultados deben estar presentes en un informe final.

\section{Recomendaciones}

\section{Infraestructura y proceso de aprendizaje}

\section{Infraestructura}

Respecto a la infraestructura específicamente se recomienda, que el laboratorio de cómputo y especializado estén juntos y con conectividad entre ellos, esto le permite al estudiante llevar a cabo un proceso definido, en el que se desarrolla la simulación y ejecución del diseño para su posterior validación. Esta infraestructura debe tener una capacidad para no más de doce personas, con condiciones ergonómicas y aptas para el aprendizaje, considerando la capacidad del docente tanto para la atención de dudas, la guía durante el proceso y la evaluación del desenvolvimiento en las diversas habilidades por parte del estudiante.

\section{Estrategias de aprendizaje}

Con la práctica de la teoría que se ejecuta en el laboratorio, el estudiante tiene la oportunidad de expresar de forma concreta los conocimientos que ha adquirido, por lo que es en este momento que tiene la oportunidad de cometer errores. Éstos también le dejarán una enseñanza y es en este momento que puede desarrollar también diversas habilidades que le serán útiles en su futuro como profesional. Es destacable en este punto, que con esta simulación se concibe de una forma más palpable conceptos como el de los costos de producción, así como de los cambios que como ingenieros se proponen, cada uno de estos conlleva una inversión o un gasto, si los mismos no tienen el éxito que se espera. El laboratorio le permite al estudiante hacer la simulación de todas las variaciones que desee en el proceso, sin tener que incurrir en ningún gasto, lo que le permite evaluar de forma integral todas las decisiones que tome en función de la mejora del proceso.

\section{Software}

La selección del equipamiento debe ir en función a la temática que se pueda desarrollar con el mismo al igual que los diversos software a utilizar, por ejemplo, para temáticas como regresión lineal, utilizar software como Minitab y para temáticas como programación lineal un software como el mencionado: Lindo o Lingo. Con esto se comprende que lo principal a considerar es la práctica de la temática que el equipo va a permitir y no ver la utilización del software 
como un fin en sí mismo, de esta forma se le demuestra al estudiante que debe desarrollar la capacidad para resolver problemas y parte de esto es investigar respecto a los software que le permitan llegar a una solución.

\section{Referencias}

Espinosa-Ríos, E A; González-López, K D; HernándezRamírez, L T; (2016). Las prácticas de laboratorio: una estrategia didáctica en la construcción de conocimiento científico escolar. Entramado, 12() 266281. Recuperado de http://www.redalyc.org/articulo. oa? $\mathrm{id}=265447025017$

Knowlton, D.S. \& Sharp, D.C. (Eds.). (2003). Problembased learning in the information age (pp. 5-44 and 81-87). San Francisco: Jossey Bass.

López, I; Nieto, R; Rodríguez, J; González, C y Jiménez, A. (2011). Clases prácticas: Una herramienta esencial en la enseñanza de las ingenierías en el marco del Espacio Europeo de Educación Superior. Teoría y Modelo Educativos, 86 (5), 523-530.

Lugo, G. (2006). Importancia de los laboratorios. Recuperado de: http://www.imcyc.com/revistact06/ dic06/INGENIERIA.pdf
Medina, A. L., Saba, G. H., Silva, J. H., \& de Guevara Durán, E. L. (2011). Los Laboratorios Virtuales y Laboratorios Remotos en la Enseñanza de la Ingeniería. Revista Internacional De Educación En Ingeniería, 4(1), 24-30.

Ortega, C. (2000). La investigación tecnológica en la Universidad del Estado de México. Recuperado de: https://books.google.co.cr/books?id=iQk_dWT_H08 $\mathrm{C} \& \mathrm{pg}=\mathrm{PA} 78 \& \mathrm{dq}=$ definici $\% \mathrm{C} 3 \% \mathrm{~B} 3 \mathrm{n}+\mathrm{de}+\mathrm{la}+$ laborat orio $\& \mathrm{hl}=\mathrm{es} \& \mathrm{sa}=\mathrm{X} \&$ redir_esc $=\mathrm{y} \# \mathrm{v}=$ onepage $\& \mathrm{q}=\mathrm{defi}$ nici\%C3\%B3n\%20de\%20la\%20laboratorio $\& \mathrm{f}=$ false

http://www.imcyc.com/revistact $06 /$ dic06/ INGENIERIA.pdf

Rosales, E. (2011). Análisis de competencias en el ejercicio profesional del ingeniero industrial, por la industria manufacturera de León, Guanajuato. Revista de la ingeniería industrial. 5(1), 132-139.

UNED. (2013). Plan de estudios carrera de Ingeniería industrial. Escuela de Ciencias Exactas y Naturales.

UNED. (s.f.). Glosario de términos curriculares para la Universidad Estatal a Distancia. Programa de apoyo curricular y evaluación de los aprendizajes. (PACE) 